\title{
Ocorrência de Haemagogus (Conopostegus) leucocelaenus (Dyar \& Shannon) e Toxorhynchites (Lynchiella) theobaldi (Dyar \& Knab) em ocos de árvore em capão de mata, Curitiba, Paraná, Brasil ${ }^{1}$
}

\author{
Mario Antonio Navarro da Silva ${ }^{2}$ \\ Ana Leuch Lozovei ${ }^{3}$
}

\begin{abstract}
Haemagogus (Conopostegus) leucocelaenus (Dyar \& Shannon) and Toxorhynchites (Lynchiella) theobaldi (Dyar \& Knab) frequency in tree holes, in a forest at Curitiba, Paraná, Brasil. The activities were performed at a preserved forest named "Capão da Imbuia", in the urban area of Curitiba, Paraná, Brazil, during march/1990 till march march/1991. The tree holes studied, showed different features: soil distance, area and volume. 303 immatures forms from seven specie of Culicidae were caught: Haemagogus (Conopostegus) leucocelaenus (Dyar \& Shannon, 1924), Culex (Culex) acharistus Root, 1927, Toxorhynchites (Lynchiella) theobaldi (Dyar \& Knab, 1906), Culex (Culex) eduardoi Casal \& Garcia, 1968, Culex (Culex) quinquefasciatus Say, 1823, Aedes (Protomacleaya) terrens (Walker, 1856). Two species were the most frequent: Haemagogus (C.) leucocelaenus and Toxorhynchites (L.) theobaldi, both of them had the higlest medias during spring and summer. These species were in overposition during the caughts.
\end{abstract}

KEY WORDS. Culicidae, Haemagogus leucocelaenus, Toxorhynchites theobaldi, tree holes

Ocos em árvore em condições de armazenar água foram considerados por JENKINS \& CARPENTER (1946), como sendo provavelmente, o mais antigo criadouro natural estabelecido para culicídeos. No entanto, de acordo com BONNET \& CHAPMAN (1956), este tipo de criadouro é considerado como fonte de menor importância, devido à reduzida produção de mosquitos, comparada com outros tipos de criadouros da referida entomofauna.

A comunidade viva neste tipo de criadouro dendrotélmico é influenciada predominantemente pelas transformações biológicas da matéria orgânica morta em biomassa microbiana, por insetos e por distúrbios esporádicos, representados por chuvas (FISH \& CARPENTER 1982; CARPENTER 1983; PARKER 1983; KITCHING 1987; WALKER et al. 1991).

Em criadouros considerados naturais, a diversidade de espécies é mais elevada em comparação com ambientes recentemente criados, como o caso dos criadouros artificiais, embora nestes a tendência seja apresentar elevada densidade de imaturos de culicídeos (AMERASINGHE \& MUNASINGHA 1988).

1) Contribuição número 1076 do Departamento de Zoologia, Universidade Federal do Paraná.

2) Departamento de Zoologia, Universidade Federal do Paraná. Caixa Postal 19020, 81531-990 Curitiba, Paraná, Brasil.

E-mail: mnavarro@bio.ufpr.br

3) Departamento de Patologia Básica, Universidade Federal do Paraná. Caixa Postal 19031, 81531-990 Curitiba, Paraná, Brasil. 
Espécies de culicídeos que normalmente utilizam como criadouro oco de árvore podem utilizar pneus com sucesso, chegando como Aedes triseriatus (Say, 1823) a abreviar o tempo de desenvolvimento no recipiente artificial (BAUGARTNER 1988). Neste caso, alguns elementos concorrem para a similaridade destes dois tipos de criadouros. A semelhança entre a estrutura da comunidade dos culicídeos nos ocos de árvores e pneus, como observado por BEIER et al. (1983). Proximidade quanto às mudanças de temperatura observada em pneus, submetidos ao sombreamento propiciado por árvores e ocos de árvore e, também, semelhança quanto à entrada de nutrientes, estando ambos os criadouros na dependência da queda de folhas, sobre as quais os fungos saprófitos irão desenvolver-se (HARAMIS 1984).

O encontro de espécie em pneu, que originariamente habita oco de árvore, foi registrado por CHADEE (1983), que fez a primeira nota de ocorrência de Haemagogus celeste Dyar \& Nuñes Tovar, 1926 em pneu, espécie esta que desempenha papel importante na transmissão silvestre do vírus da febre amarela na América Neotropical. No Brasil GoMES \& MARQUES (1988) coletaram imaturos de Aedes albopictus Skuse, 1894 em ocos de árvore Flambuiã (Delonix regia (Hook.) Raf.), destacando a importância epidemiológica deste encontro, uma vez que caracteriza hábito silvestre desta espécie importada, que já havia sido encontrada em recipiente artificial, como apontado por BRITO et al. (1986).

A proximidade ecológica entre criadouros naturais e artificiais, a possibilidade de aproximação física entre estes tipos de criadouros, em conseqüência da existência de áreas de mata preservada inseridas em região urbana, aliada à oferta em número superior de criadouros artificiais e à importância epidemiológica de certas espécies que utilizam preferencialmente ocos de árvore, determinou como objetivo deste trabalho o estudo de ocos de árvore em área de mata preservada no Município de Curitiba, Paraná.

\section{MATERIAL E MÉTODOS}

O trabalho foi desenvolvido na área urbana do município de Curitiba, estado do Paraná, localizado na região centro-sul do Primeiro Planalto do Estado do Paraná, e região oriental do Estado, estendendo-se do norte ao sul, em forma de arco, com uma largura de 70 a $80 \mathrm{~km}$, limitando-se a leste com os Municípios de Piraquara e Pinhais e a oeste com o Município de Campo Largo, nas coordenadas $25^{\circ} 25^{\prime} 04^{\prime \prime S}$ e 49 $14^{\prime} 30^{\prime \prime} \mathrm{W}$, a uma altitude de $945 \mathrm{~m}$. Em relação ao aspecto climático, a cidade apresenta temperatura média anual igual a $16,5^{\circ} \mathrm{C}$, com média anual de precipitação pluviométrica de $1.451,8 \mathrm{~mm}$ e média anual de umidade relativa do ar igual a $81,5 \%$ (MAACK 1981).

Para o desenvolvimento das atividades de campo foi escolhida a área do Museu de História Natural do Capão da Imbuia, situado em bairro homônimo da cidade de Curitiba, a $10.000 \mathrm{~m}$ a leste do marco zero da cidade, contando com área de aproximadamente $32.000 \mathrm{~m}^{2}$. Esta área é recoberta por vegetação semelhante a que recobria esta região do Paraná, estando, no entanto, incluída na área urbana. Atualmente esta reserva de mata encontra-se totalmente circundada pela estrutura urbana. Informações mais detalhadas sobre a flora do Capão da Imbuia foram descritas em SiLva \& LOZOVEI (1996). 
Para a realização de coleta de imaturos de Culicidae em ocos de árvore, a área do Capão da Imbuia foi dividida em dois blocos A e B. As coletas foram realizadas quinzenalmente, de maneira alternada entre as áreas A e B. Com este procedimento, os criadouros dendrícolas de cada bloco eram investigados com periodicidade mensal, durante o período de março de 1990 a março de 1991.

Como primeiro procedimento, foi realizado levantamento dos ocos de árvores em cada um dos blocos, que atingiam no máximo 3 metros do nível solo. Uma vez localizados os ocos de árvore, foi determinada a capacidade volumétrica máxima, preenchendo a cavidade com água destilada até o limite de transbordamento, concomitantemente introduzida uma régua até a parte mais profunda, com o objetivo de determinar a profundidade máxima dos ocos.

Foram ainda determinadas a altura dos ocos de árvores em relação ao nível do solo e o raio máximo da abertura dos mesmos. Em cada coleta foram pesquisados quatro ocos de árvores. A água dos ocos foi retirada pelo processo de sifonagem como descrito por (FORATTINI 1962). Após o esgotamento da água, procedia-se a lavagem das paredes dos ocos, com água de poço artesiano. Toda água assim retirada, foi passada através de peneira de malha de $200 \mu \mathrm{m}$ de diâmetro. O material retido na peneira era acondicionado em frascos de transporte com capacidade de $250 \mathrm{ml}$. O aspecto geral dos ocos investigados no Capão da Imbuia está representado na figura 1 .

Parte dos espécimens identificados está depositada na "Coleção Pe. Jesus Santiago Moure" do Departamento de Zoologia de Universidade Federal do Paraná.

\section{RESULTADOS}

Durante o período de estudo foram coletados 303 imaturos pertencentes a sete espécies de Culicidae. As espécies encontradas foram separadas em dois grupos de acordo com a freqüência de ocorrência. O primeiro, composto por Haemagogus (C.) leucocelaenus (Dyar \& Shannon, 1924) e Toxorhynchites (L.) theobaldi (Dyar \& Knab, 1906), espécies coletadas regularmente durante o período analisado, em respectivamente $51,85 \%$ e $62,96 \%$ da coletas, e o segundo grupo, formado por Culex acharistus Root, 1927, Culex eduardoi Casal \& Garcia, 1968, Culex quinquefasciatus Say, 1823 e Aedes terrens (Walker, 1856) que ocorreram de forma esporádica (Tab. I).

Tabela I. Número total e porcentagem de culicídeos imaturos coletados em ocos de árvore, de março de 1990 a março de 1991, na área do Capão da Imbua, Curitiba, Paraná.

\begin{tabular}{lcc}
\hline \multicolumn{1}{c}{ Espécies } & Número & Porcentagem \\
\hline Haemagogus (Conopostegus) leucocelaenus & 118 & 38,94 \\
Culex (Culex) acharistus & 105 & 34,65 \\
Toxorhynchites (Lynchiella) theobaldi & 44 & 14,53 \\
Culex (Culex) eduardoi & 15 & 4,95 \\
Culex (Culex) quinquefasciatus & 13 & 4,29 \\
Aedes (Protomacleaya) terrens & 8 & 2,64 \\
\hline Total & 303 & 100,00 \\
\hline
\end{tabular}




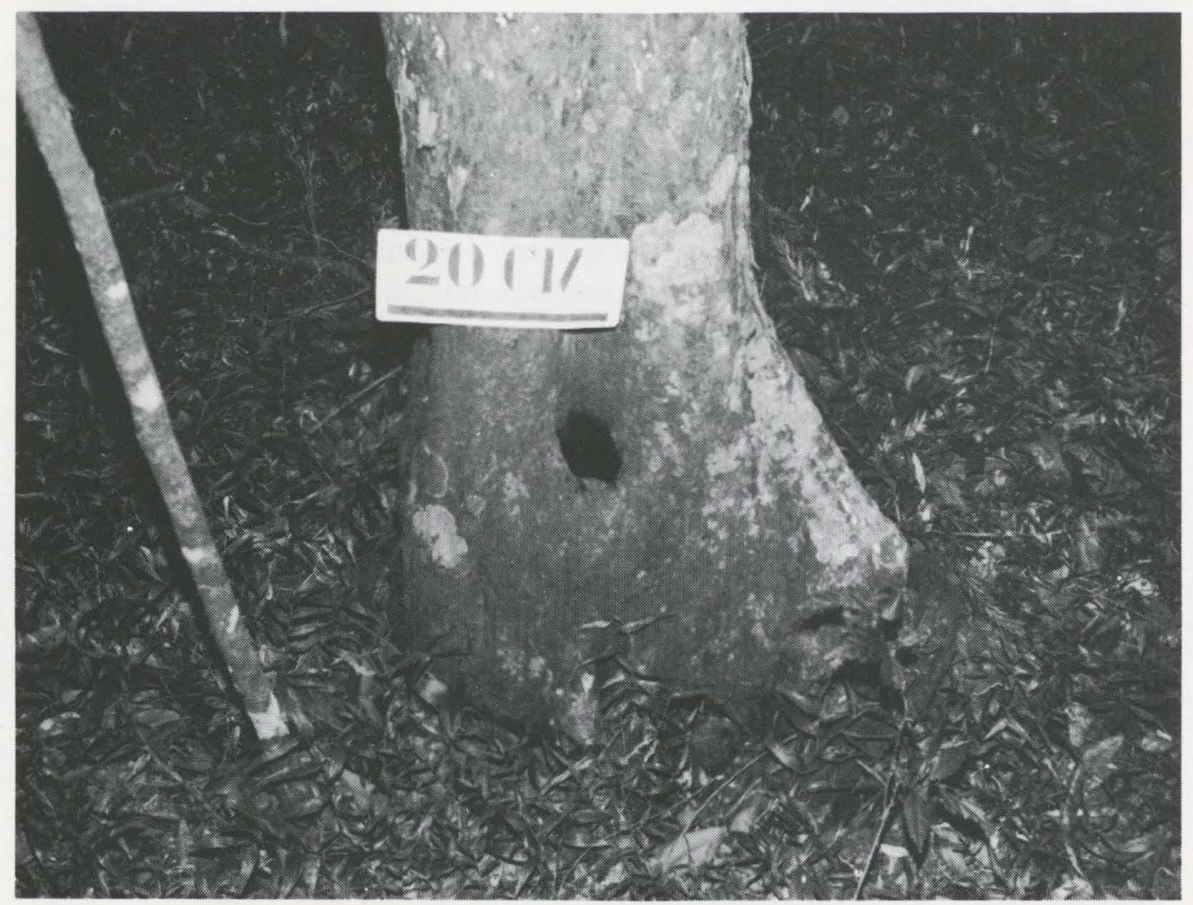

Fig. 1. Aspecto de oco de árvore investigado no interior da mata do Capão da Imbuia, Curitiba, Paraná, Brasil.

Os ocos de árvore analisados no Capão da Imbuia foram agrupados em classes de acordo com o volume, altura do solo, raio de abertura do oco e profundidade. Os dois primeiros fatores analisados, volume e altura do solo, apresentaram elevada oscilação. Em relação ao volume, foram investigados ocos com capacidade de $25,0 \mathrm{ml}$ até $4875,0 \mathrm{ml}$; este último volume pertencente à escavação em tronco caído. Na tabela II, são apresentadas as características dos ocos estudados.

Tabela II. Número de ocos de árvores analisados, de março de 1990 a março de 1991, no Capão da Imbuia, Curitiba, Paraná. Agrupados em classes segundo a capacidade volumétrica, altura do nível do solo, raio da boca e profundidade.

\begin{tabular}{|c|c|c|c|c|c|c|c|}
\hline \multicolumn{2}{|c|}{ Volume (ml) } & \multicolumn{2}{|c|}{ Altura $(\mathrm{cm})$} & \multicolumn{2}{|c|}{ Raio da boca $(\mathrm{cm})$} & \multicolumn{2}{|c|}{ Profundidade $(\mathrm{cm})$} \\
\hline Classes & Número & Classes & Número & Classes & Número & Classes & Número \\
\hline $1-99$ & 2 & $1-49$ & 5 & $4-9$ & 7 & $4-9$ & 16 \\
\hline $100-199$ & 3 & $50-99$ & 9 & $10-15$ & 11 & $10-15$ & 3 \\
\hline $200-299$ & 5 & $100-200$ & 4 & $16-20$ & 1 & $>15$ & 1 \\
\hline $300-399$ & 4 & $>200$ & 2 & $>20$ & 1 & & \\
\hline $400-499$ & 3 & & & & & & \\
\hline$>500$ & 3 & & & & & & \\
\hline Total & 20 & & 20 & & 20 & & 20 \\
\hline
\end{tabular}


Dentre as espécies que ocorreram de forma esporádica, destacamos Culex acharistus, da qual foram coletados 105 imaturos explorando microabitat que comportava o volume de água mais elevado, $4875,0 \mathrm{ml}$. Também Culex eduardo $i$ e Aedes terrens, espécies que foram coletadas explorando simultaneamente ocos de árvore com Haemagogus leucocelaenus, sendo que desta espécie, no primeiro caso, foi coletado um imaturo e no segundo caso, três.

Durante o ano de realização das coletas, foram registradas pequenas diferenças entre os níveis de precipitação pluviométrica nas quatro estações. Já em relação à temperatura, como esperado para o Município de Curitiba, houve quedas acentuadas durante outono e inverno (Tab. III). Desta forma, as populações de Haemagogus leucocelaenus e Toxorhynchites theobaldi estavam sujeitas principalmente às oscilações da temperatura durante o período analisado. Ocorreu predomínio de ambas espécies na primavera e verão (Fig. 2).

Tabela III. Média estacional e desvio padrão da temperatura máxima e mínima e somatória da precipitação pluviométrica para igual intervalo, durante o período de março de 1990 a março de 1991 em Curitiba, Paraná.

\begin{tabular}{|c|c|c|c|}
\hline \multirow{2}{*}{ Estaçర̄es } & Temperatura máxima $\left({ }^{\circ} \mathrm{C}\right)$ & Temperatura mínima $\left({ }^{\circ} \mathrm{C}\right)$ & Precipitaçăo pluviométrica (mm) \\
\hline & Média & Média & Somatória \\
\hline Outono & $22,05 \pm 3,77$ & $12,46 \pm 4,72$ & 381,70 \\
\hline Inverno & $18,68 \pm 4,74$ & $8,21 \pm 3,79$ & 448,60 \\
\hline Primavera & $24,40 \pm 4,68$ & $14,98 \pm 3,00$ & 404,60 \\
\hline Verăo & $26,02 \pm 3,08$ & $16,14 \pm 2,11$ & 418,50 \\
\hline
\end{tabular}

Haemagogus leucocelaenus somente não foi coletada durante os meses de maio a setembro. Neste período, a temperatura média mensal mínima do ar oscilou entre $7,8^{\circ} \mathrm{C}$ e $9,4^{\circ} \mathrm{C}$. No entanto, no mês de abril ainda coletou-se imaturos de $T$. theobaldi com temperatura média mínima alcançando $5,9^{\circ} \mathrm{C}$ e máxima de $24,5^{\circ} \mathrm{C}$.

Nos ocos de árvore, nos quais foram coletados exclusivamente imaturos de H. leucocelaenus ou Toxorhynchites theobaldi, a capacidade máxima de retenção de água oscilou entre $110 \mathrm{ml}$ até $1575 \mathrm{ml}$. Para a segunda espécie, a média do raio da boca do oco de árvore foi igual a $11,78 \mathrm{~cm}$ com coleta em média de 1,78 larvas por oco de árvore investigado.

No mesmo microabitat $H$. leucocelaenus e $T$. theobaldi foram coletados em sete oportunidades, em ocos com capacidade de retenção de água oscilando entre $295 \mathrm{ml}$ até $445 \mathrm{ml}$, intervalo inferior ao registrado para ocorrência solitária de ambas espécies. Quanto à altura, estes ocos encontravam-se entre $33,0 \mathrm{~cm}$ a $99,0 \mathrm{~cm}$ com média do raio de abertura da boca igual a $10,7 \mathrm{~cm}$. O número máximo de larvas de T. theobaldi coletado nestes ocos foi de duas. Por outro lado, foram encontradas de uma a até 19 larvas de $H$. leucocelaenus coabitando com T. theobaldi.

Do grupo das espécies de ocorrência esporádica, Culex acharistus e Culex eduardoi tiveram sua ocorrência registrada no mesmo microabitat. Em tronco de árvore caída cuja escavação comportava aproximadamente $4875 \mathrm{ml}$ de água, com profundidade de $13 \mathrm{~cm}$, altura do solo de $66,0 \mathrm{~cm}$ e diâmetro da concavidade de 
$57,5 \mathrm{~cm}$, neste mesmo microabitat foram coletados os imaturos de Culex eduardoi. Imaturos de Aedes terrens e Culex quinquefasciatus foram coletados em diferentes oportunidades, em oco de árvore com as características de capacidade volumétrica para $1575 \mathrm{ml}$ de água, profundidade de $15 \mathrm{~cm}$, diâmetro da boca igual a $18 \mathrm{~cm} \mathrm{e}$ altura em relação ao solo de $13 \mathrm{~cm}$.

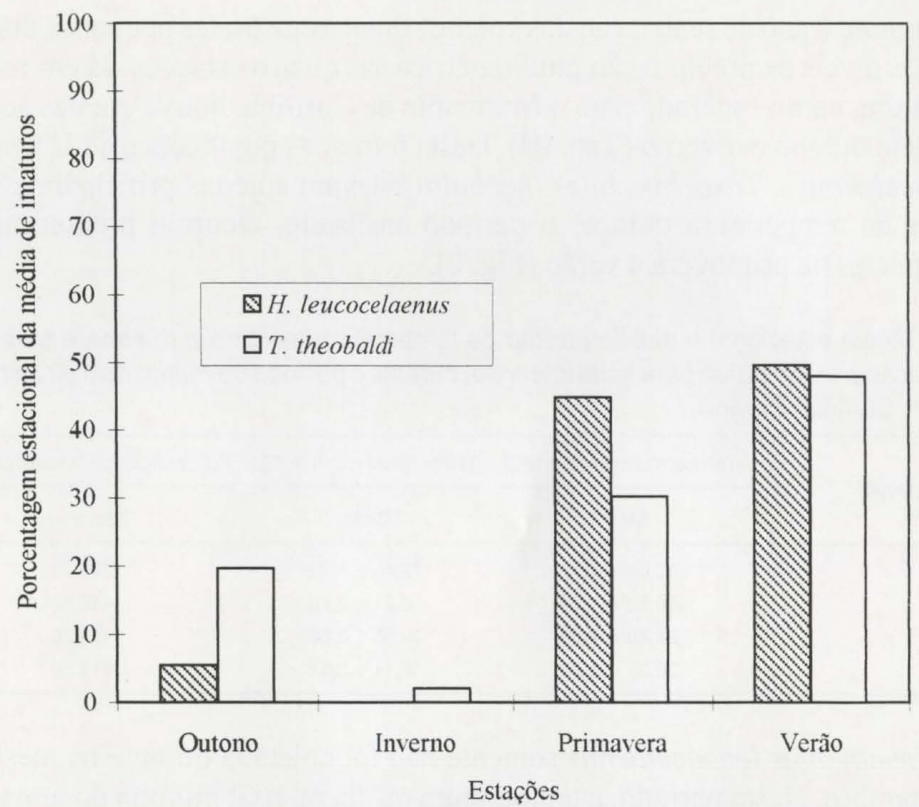

Fig. 2. Porcentagem estacional da média de imaturos de $H$. leucocelaenus e $T$. theobaldi, coletados no período de março de 1990 a março de 1991, no Capão da Imbuia, Curitiba, Paraná, Brasil.

\section{DISCUSSÃO}

A investigação de ocos de árvores em capão de mata na área urbana permitiu detectar a presença de espécies amplamente adaptadas ao ambiente urbano e outras com maior proximidade ao ambiente silvestre.

O encontro de espécimens de Haemagogus leucocelaenus reveste-se de grande interesse epidemiológico, em conseqüência de ser considerada vetor potencial do vírus da febre amarela pelos autores SHANNON et al. (1938), CERQUEIRA (1961), PINHEIRO et al. (1981), HERVÉ \& ROSA (1983), GUIMARÃES \& ARLÉ (1984), HERVÉ et al. (1985), FORATTINI et al. (1986); sendo ainda considerada possuidora de caráter silvestre podendo ser encontrada em manchas residuais de mata que lhe podem servir de abrigo, ForATTINI \& GOMES (1988). Apresenta como criadouro natural ocos de árvore conforme caracterizados por DAVID (1944), GALINDO et al. (1955), CERQUEIRA (1961), ForATTINI et al. (1968) e GOMES et al. (1992). 
Haemagogus leucocelaenus apresentou grande atividade nos meses mais quentes e chuvosos, porém, sob condições de longos períodos de seca, pode não ser ativa ou não sobreviver (FoRATTINI \& GOMES 1988; CHADEE \& ELISHA 1989). As condições climáticas em Curitiba, no período analisado, apresentaram precipitação pluviométrica uniforme sem períodos que possam ser considerados secos. O período de temperaturas mais elevadas, no entanto, ficou praticamente limitado à primavera e ao verão, período de ocorrência de aproximadamente noventa e cinco porcento dos imaturos coletados. Isso pode indicar para Curitiba a temperatura como fator ambiental limitante para distribuição quantitativamente homogênea ao longo dos diferentes períodos sazonais.

A outra espécie capturada com freqüência, Toxorhynchites theobaldi, possui ampla distribuição desde América Central até o Brasil, sendo considerada predador passivo no estágio larval sob condições de laboratório, com as fêmeas realizando oviposição em criadouros naturais e artificiais (RUBIO et al. 1980; CASTER \& BAILEY 1984). Em conseqüência do hábito alimentar, imaturos de Toxorhynchites sp. foram utilizados para controle das populações de outras espécies de Culicidae por GOMA (1964), GERBER \& VISSER (1978), FOCKS et al. (1982), BAILEY et al. (1983a,b), entre outros. Além da predação de larvas de outras espécies de culicídeos, pode também ocorrer canibalismo principalmente durante os últimos ínstares larvais (CASTER \& BAILEY 1984).

A utilização de recipientes artificiais localizados em área sombreada por Toxorhynchites theobaldi foi assinalada como preferencial por RUBIO \& AYESTA (1984), REYes-VILlanUeVA et al. (1987) e RodRIGUEZ \& REYES-VILlaNEUVA (1992). Em criadouros naturais como ocos de árvore, esta espécie apresentou resistência às oscilações do nível de água, estando adaptada a ambientes secos (RUSSO 1986). Com a distribuição uniforme das chuvas e com a adaptação às condições de seca desta espécie, este fator meteorológico não pode ser indicado como limitante durante o período analisado.

O número de ovos colocados pelas fêmeas de Toxorhynchites sp. em criadouros naturais, como ocos de árvore, apresentou relação diretamente proporcional à superfície disponível para oviposição e à taxa de oviposição das presas (RUSSO 1986; ARREDONDO-BERNAL \& REYES-VILLANUEVA 1989).

Os ocos de árvores investigados no Capão da Imbuia apresentaram, em média, reduzida superficie para oviposição, o que pode ter influenciado no reduzido número de larvas de $T$. theobaldi coletadas. Nos ocos investigados os imaturos de H. leucocelaenus podem ser considerados como a principal presa para imaturos de T. theobaldi, uma vez que estas espécies foram encontradas em superposição com relativa freqüência. A exemplo da espécie supra citada, a sua maior ocorrência é influenciada pelas temperaturas mais elevadas.

As três espécies menos freqüentes coletadas nos ocos apresentaram comportamento similar quanto ao tipo de habitat explorado. Neste mesmo capão de mata, Silva \& LozoveI (1996) coletaram de forma esporádica Culex acharistus e Culex eduardoi em recipientes artificiais introduzidos no interior da mata. FORATTINI (1962) fez referência a estas espécies como pouco conhecidas, as quais tiveram preferência por grandes criadouros, como pântanos. Já LOPES (1997) encontrou preferência significativa de Culex eduardoi por pneus. 
Imaturos de Aedes terrens foram coletados em única oportunidade durante o verão em oco de árvore. Este tipo de habitat para esta espécie já havia sido assinalado por DAVID (1944), GALINDO et al. (1955), NEVES \& FARIA (1977), LOURENÇO-DE-OLIVEIRA et al. (1986). Esta espécie ainda foi coletada por GOMES et al. (1992) em composição com Haemagogus leucocelaenus e Toxorhynchites sp.

DEGALLIER et al. (1978), na Guiana Francesa, consideraram Aedes terrens estritamente florestal. Já GUIMARÃES et al. (1989), em áreas da mata atlântica no município de Itaguaí, Rio de Janeiro, capturaram exemplares daquela espécie no interior da mata.

No Capão da Imbuia detectou-se a presença de espécies que utilizam desde o ambiente de mata até o domicílio. Se houver capacidade reprodutiva da espécie e oferta suficiente gerada pela atividade antrópica, a potencialidade de utilização de amplo espectro de ambientes, a proximidade física de habitats com características contrastantes, como área de mata e região urbana, bem como a similaridade ecológica de certos criadouros naturais e artificiais, pode levar espécies que preferencialmente utilizam criadouros naturais ao deslocamento para criadouros artificiais.

AGRADECIMENTOS. Ao Prof. Dr. Oswaldo Paulo Forattini da Faculdade de Saúde Pública da Universidade de São Paulo pela confirmação da identificação específica dos Culicidae.

\section{REFERÊNCIAS BIBLIOGRÁFICAS}

AMERASINGHE, F.P. \& N.B. MunASINGHA. 1988. A predevelopment mosquito survey in the Mahaweli development Project Area, Sri Lanka: Immatures. Jour. Med. Entomol. 25 (4): 286-294.

ARredondo-BERnal, H.C. \& F. ReYes-VilanueVa. 1989. Diurnal pattern and behavior of oviposition of Toxorhynchites theobaldi in the field. Jour. Amer. Mosq. Control Assoc. 5 (1): 25-28.

BAILEY, D.L.; D. ADABBE; D.A. FocKS \& D.A. DAME. 1983a. Field releases with adults and pupae of Toxorhynchites rutilus rutilus (Diptera:Culicidae). Jour. Med. Entomol. 20 (3): 308-312.

BAILEY, D.L.; R.G. JonES \& P.R. Simmonos. 1983b. Effects of indigenous Toxorhynchites rutilus rutilus on Aedes aegypti breeding in tire dumps. Mosq. News. 43 (1): 33-37.

BAUMGARTNER, D.L. 1988. Suburban accumulations of discarded tires in northeastern Illinois and their associated mosquitoes. Jour. Amer. Mosq. Control Assoc. 4 (4): 500-508.

Beier, J.C.; N. Travis; C.E. Patricoski \& J. KranzFelder. 1983. Habitat segregation among larval mosquitoes (Diptera: Culicidae) in tire yards in Indiana, USA. Jour. Med. Entomol. 20 (1): 76-80.

BONNET, D.D. \& H. CHAPMAN. 1956. The importance of mosquito breeding in tree holes with special reference to the problem in Tahiti. Mosq. News. 16 (4): 301-305.

Brito, M. DE; G.R.A.M. Marques; C.C.A. Marques \& R.M. TubaKi. 1986. 
Primeiro encontro de Aedes (Stegomyia) albopictus (Skuse) no Estado de São Paulo (Brasil). Rev. Saúde Públ., São Paulo, 20 (6): 489.

CASTER, J.L. \& D.L. BAILEY. 1984. Effects of Toxorhynchites amboinensis on laboratory reared Aedes aegypti populations (Diptera: Culicidae). Jour. Med. Entomol. 21 (2): 133-136.

CARPENTER, S.R. 1983. Resource limitation of larval tree-hole mosquitoes subsisting on beech detritus. Ecology 64: 219-223.

CERQueIRA, N.L. 1961. Distribuição geográfica dos mosquitos da Amazônia. Revta bras. Ent. 10: 111-168.

CHADEE, D.D. 1983. Rock hole breeding Haemagogus mosquitoes on Monos Island, Trinidad, West Indies. Mosq. News. 43 (2): 236-237.

ChadeE, D.D. \& S.T. ElishA. 1989. Observations on the seasonal incidence and diel oviposition periodicity of Haemagogus mosquitoes (Diptera: Culicidae) in Trinidad, W.I.: Part I. Haemagogus janthinomys Dyar. Ann. Trop. Med. Parasitol. 83 (5): 507-516.

DAvID, E.D. 1944. Larval habitats of some Brazilian mosquitoes. Rev. Ent., Rio de Janeiro, 15 (1-2): 221-235.

DeGallier, N.; X.F. Pajot; R. Kramer; J. Claustre; S. Bellong \& F. Le Pont. 1978. Rythmes d'activité des Culicidés de la Guyane Française (Diptera: Culicidae). Cash. O.R.S.T.O.M. Sér. Ent. Méd. Parasitol. 16: 173-84.

FISH, D. \& S.R. CARPENTER. 1982. Leaf litter and larval mosquito dynamics in tree-hole ecosystems. Ecology 63 (2): 283-288.

FoCKS, D.A.; S.R. SACKETT \& D.L. BAILEY. 1982. Field experiments on the control of Aedes aegypti and Culex quinquefasciatus by Toxorhynchites rutilus rutilus (Diptera: Culicidae). Jour. Med. Entomol. 19 (3): 336-339.

ForATTINI, O.P. 1962. Entomologia Médica. São Paulo, Ed. Universidade de São Paulo, Vol. 1, 662p.

Forattini, O.P. \& A. DE C. GoMEs. 1988 Biting activity of Aedes scapularis (RONDANI) and Haemagogus mosquitoes in southern Brazil (Diptera: Culicidae). Rev. Saúde Públ., São Paulo, 22 (2): 84-93.

ForatTINI, O.P.; A. DE C. GoMES; D. NATAL \& J.L.F. SANTOS. 1986. Observações sobre atividade de mosquitos Culicidae em mata primitiva da encosta no Vale do Ribeira, São Paulo, Brasil. Rev. Saúde Públ., São Paulo, 20 (1): 1-20.

FoRATTINI, O.P.; O.D.S. LOPES \& E.X. RABELLO. 1968. Investigações sobre o comportamento de formas adultas de mosquitos silvestres no Estado de São Paulo, Brasil. Rev. Saúde Públ., São Paulo, 2 (2): 111-173.

GALINDO, P.; S.J. CARPENTER \& H. TRAPIDO. 1955. A contribution to the ecology and biology of tree hole breeding mosquitoes of Panama. Ann. Entomol. Soc. Amer. 48: 158-164.

GERBERG, E.J. \& W.M. VisSER. 1978. Preliminary field trial for the biological control of Aedes aegypti by means of Toxorhynchites brevipalpis, a predatory mosquito larva. Mosq. News. 38 (2): 197-200.

GoMA, L.K.H. 1964. Laboratory observations on the influence of illumination on the predatory habits of Toxorhynchites larvae (Diptera, Culicidae). Ann. trop. Med. Parasitol. 58: 350-354.

Gomes, A. DE C. \& G.R.A. MARQuES. 1988. Encontro de criadouro natural de 
Aedes (Stegomyia) albopictus (Skuse). Estado de São Paulo, Brasil. Rev. Saúde Públ., São Paulo, 22 (3): 245.

Gomes, A. DE C.; O.P. ForatTini; I. KAKITANI; G.R.A.M. MARQUES; C.C. DE A. MARQUeS; D. MARUCCI \& M. DE BRITO. 1992. Microhabitats de Aedes albopictus (Skuse) na região do Vale do Paraíba, Estado de São Paulo, Brasil. Rev. Saúde Públ., São Paulo, 26 (2): 108-118.

Guimarães, A.E. \& M. ArLé. 1984. Mosquito no Parque Nacional da Serra dos Órgãos, Estado do Rio de Janeiro, Brasil. I. Distribuição estacional. Mem. Inst. Oswaldo Cruz. 79 (3): 309-323.

Guimarães, A.E.; M.A. MotTA; M. Arlé; R.M. Machado; L.D. GonÇALves. 1989. Bionomia de mosquitos (Diptera: Culicidae) em áreas da Mata Atlântica no Munícipio de Itaguaí, Estado do Rio de Janeiro, Brasil. I. Freqüência intra, peri e extradomiciliar. Mem. Inst. Oswaldo Cruz. 84 (supl.IV): 243-254.

HARAMIS, L.D. 1984. Aedes triseriatus: A comparison of density in tree holes vs. discarded tires. Mosq. News. 44 (4): 485-489.

HERVÉ, Jean-Pierre \& A.P. T. da ROSA. 1983. Ecologia da febre amarela no Brasil. Rev. Fundação Sesp, São Paulo, 28 (1): 11-19.

HeRvÉ, J.P.; G.C. SÁ FiLHO; A.P.A.T. DA RosA \& N. DÉGALlier. 1985. Bio-écologie d'Haemagogus (Haemagogus) janthinomys Dyar au Brésil. Établissement du cycle gonotrophique en laboratoire et estimation du taux de survie. Cash. O.R.S.T.O.M. Sér. Ent. Méd. Parasitol. 23 (3): 203-208.

JENKINS, D.W. \& S.J. CARPENTER. 1946. Ecology of the tree hole breeding mosquitoes of Neartic North America. Ecol. Monogr. 16: 31-47.

KITCHING, R.L. 1987. Spatial and temporal variation in food webs in water-filled tree holes. Oikos 48: 280-288.

LOPES, J. 1997. Ecologia de mosquitos (Diptera, Culicidae) em criadouros naturais e artificiais de área rural do norte do Estado do Paraná, Brasil. VI. Coletas de larvas no peridomicílio. Revta bras. Zool. 14 (3): 571-578.

LOURENÇO-DE-OliveIRA, R.; R. HEYDEN \& T.F. Silva. 1986. Alguns aspectos da ecologia dos mosquitos (Diptera, Culicidae) de uma área de planície (Granjas, Calábria), em Jacarepaguá, Rio de Janeiro.V. Criadouros. Mem. Inst. Oswaldo Cruz. 81 (3): 265-271.

MAACK, R. 1981. Geografia Física do Estado do Paraná. São Paulo, J. Olympio, $2^{\mathrm{a}}$ ed., $450 \mathrm{p}$.

NEVES, D.P. \& A.C. DE FARIA. 1977. Biologia do Aedes (Finlaya) terrens (Walker) em condições de campo e laboratório (Diptera, Culicidae). Rev. Brasil. Biol. 37 (4): 803-806.

Pinheiro, F.P.; A.P.A. TRavassos Da Rosa; M.A.P. Moraes. 1981. An epidemic of yellow fever in Central Brazil, 1972-1973. II. Ecological studies. Amer. Jour. Trop. Med. Hyg. 30 (1): 204-211.

PARKER, G.G. 1983. Throughfall and stemflow in the forest nutrient cycle. Adv. Ecol. Res. 13: 57-133.

ReYes-Villanueva, F.; M.H. Badii; M. L. Rodriguez-Tovar \& M. VillarReAL-LEAL. 1987. Oviposition of Toxorhynchites theobaldi in different types of artificial containers in Mexico. Jour. Amer. Mosq. Control Assoc. 3 (4): 651-654. 
Rodriguez, A.D. \& F. ReYes-VillanUeva. 1992. Comportamiento sexual de Toxorhynchites theobaldi bajo condiciones de laboratório. Southwest. Entomol. 17 (3): 255-260.

RUBIO, Y.\& C. AYESTA 1984. Laboratory observations on the biology of Toxorhynchites theobaldi. Mosquito News 44 (1): 86-90.

RuBIO, Y.; D. RodRÍGUEZ; C.E. MACHADO-ALLISON \& J.A. LEÓN. 1980. Algunos aspectos del comportamiento de Toxorhynchites theobaldi (Diptera - Culicidae). Acta Cient. Venez. 31: 345-351.

Russo, R. 1986. Comparison of predatory behavior in five species of Toxorhynchites (Diptera: Culicidae). Ann. Entomol. Soc. Amer. 79 (4): 715-722.

SHANNON, R.C.; L. WHITMAN \& M. FRANCA. 1938. Yellow fever virus in jungle mosquitoes. Science 88: 110-11.

Silva, M.A.N. DA \& A.L. Lozover. 1996. Criadouros de imaturos de mosquitos (Diptera, Culicidae) introduzidos em mata preservada na área urbana de Curitiba, Paraná, Brasil. Revta bras. Zool. 13 (4): 1023-1042.

WALKER, E.D.; D.L. LANSON; R.W. MERRIT; N.T. MORGAN \& M.J. KLUG. 1991. Nutrient dynamics, bacterial populations, and mosquito productivity in tree hole ecosystems and microcosms. Ecology 72 (5): 1529-1546.

Recebido em 20.VIII.1998; aceito em 04.VI.1999. 